University of Nebraska - Lincoln

DigitalCommons@University of Nebraska - Lincoln

April 2004

\title{
Determining the Biosynthetic Sequence in the Early Steps of the Fumonisin Pathway by Use of Three Gene-Disruption Mutants of Fusarium verticillioides
}

\author{
Ravi S. Bojja \\ University of Nebraska - Lincoln \\ Ronald Cerny \\ rcerny1@unl.edu \\ Robert H. Proctor \\ National Center for Agricultural Utilization Research, Agricultural Research Service, U.S. Department of \\ Agriculture, 1815 North University Street, Peoria, Illinois \\ Liangcheng Du \\ University of Nebraska - Lincoln, Idu3@unl.edu
}

Follow this and additional works at: https://digitalcommons.unl.edu/chemistrycerny

Part of the Chemistry Commons

Bojja, Ravi S.; Cerny, Ronald; Proctor, Robert H.; and Du, Liangcheng , "Determining the Biosynthetic Sequence in the Early Steps of the Fumonisin Pathway by Use of Three Gene-Disruption Mutants of Fusarium verticillioides" (2004). Ronald Cerny Publications. 6.

https://digitalcommons.unl.edu/chemistrycerny/6

This Article is brought to you for free and open access by the Published Research - Department of Chemistry at DigitalCommons@University of Nebraska - Lincoln. It has been accepted for inclusion in Ronald Cerny Publications by an authorized administrator of DigitalCommons@University of Nebraska - Lincoln. 


\title{
Determining the Biosynthetic Sequence in the Early Steps of the Fumonisin Pathway by Use of Three Gene-Disruption Mutants of Fusarium verticillioides
}

\author{
Ravi S. Bojua,${ }^{\dagger}$ Ronald L. Cerny,${ }^{\dagger}$ Robert H. Proctor,$\stackrel{\ddagger}{\ddagger}$ And \\ LIANGCHENG DU*,† \\ Chemistry Department, University of Nebraska-Lincoln, Lincoln, Nebraska 68588-0304, and \\ National Center for Agricultural Utilization Research, Agricultural Research Service, \\ U.S. Department of Agriculture, 1815 North University Street, Peoria, Illinois 61604-3999
}

\begin{abstract}
Fumonisins are polyketide-derived mycotoxins produced by Fusarium verticillioides, a fungal pathogen of corn plants. Although a gene cluster for the biosynthesis of fumonisins has been cloned, the biosynthetic pathway is still not clear. We have used three gene-disrupted mutants, designated $\triangle F U M 1, \triangle F U M 6$, and $\triangle F U M 8$, to study the early steps of the pathway. Fumonisins were not produced in single-strain cultures of the $\triangle F U M 1, \triangle F U M 6$, and $\triangle F U M 8$ mutants. However, fumonisins were produced by $\triangle F U M 1$ or $\triangle F U M 8$ mutants when they were cocultured with the $\triangle F U M 6$ mutant. No fumonisins were produced when the $\triangle F U M 1$ and $\triangle F U M 8$ mutants were cocultured. These results suggest that the $\triangle F U M 6$ mutant produces a fumonisin intermediate that can be further metabolized by fumonisin biosynthetic enzymes in the $\triangle F U M 1$ and $\triangle F U M 8$ mutants. To isolate the potential intermediates produced by $\triangle F U M 6$, we followed a time course of cocultures of the $\triangle F U M 1$ and $\triangle F U M 6$ and the $\triangle F U M 8$ and $\triangle F U M 6$ mutants. Liquid chromatographic-mass spectrometric data suggested that metabolites having the general carbon skeleton of fumonisins with 1-4 hydroxyl groups were accumulated over a 7-day period. These results indicate that fumonisin biosynthesis starts with Fum1pcatalyzed carbon-chain assembly followed by the Fum8p-catalyzed alanine condensation. The resulting product then can be further oxidized by Fum6p and other enzymes.
\end{abstract}

KEYWORDS: Fumonisin; Fusarium verticillioides; mycotoxin; biosynthesis

\section{INTRODUCTION}

Fumonisins are polyketide-derived mycotoxins produced by the filamentous fungus Fusarium verticillioides (Sacc.) Nirenberg (synonym $F$. moniliforme, teleomorph Gibberella moniliformis, synonym $G$. fujikuroi mating population A) $(1,2)$. The fungus is a widespread pathogen of corn plants, on which it causes ear and stalk rots (3). Fumonisins cause several fatal animal diseases, including leukoencephalomalacia in horses, pulmonary edema in swine, and cancer in rats and mice $(1,4$, $5)$. In addition, fumonisins are suspected to cause human esophageal cancer (1).

Wild-type strains of $F$. verticillioides produce predominantly four $\mathrm{B}$-series fumonisins $\left(\mathrm{B}_{1}, \mathrm{~B}_{2}, \mathrm{~B}_{3}\right.$, and $\left.\mathrm{B}_{4}\right)$, with fumonisin $\mathrm{B}_{1}$ making up to approximately $70 \%$ of the total content (2). These compounds have a linear 20-carbon backbone with hydroxyl, methyl, and tricarballylic acid moieties at various positions along the backbone (Figure 1) (6). The backbone originates from a $\mathrm{C}_{18}$ polyketide chain and one amino acid.

* To whom correspondence should be addressed: telephone 402-472 2998; fax 402-472-9402; e-mail ldu@unlserve.unl.edu.

University of Nebraska-Lincoln.

$\doteqdot$ U.S. Department of Agriculture.<smiles>[R9]C(CCCCC([2H])CC(O)C(C)N)CC(C)CC(O)C(O)C(C)CCCC</smiles>

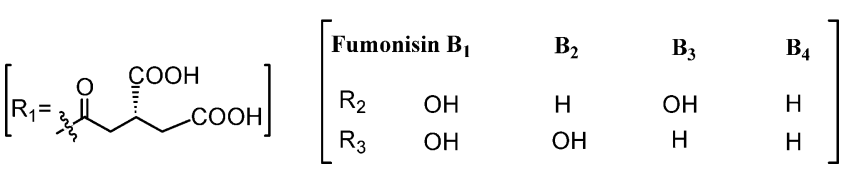

Figure 1. Chemical structures of B-series fumonisins.

Isotope feeding experiments have shown that the carbons 3-20 of the backbone are derived from acetate and that the amino group and $\mathrm{C}-1$ and $\mathrm{C}-2$ are derived from alanine $(7,8)$. The origin of several moieties attached to the backbone has also been studied. The two methyl groups on C-12 and C-16 are derived from methionine (9). The hydroxyl group on C-3 is from an acetate-derived carbonyl group, whereas the hydroxyl groups on C-5, C-10, C-14, and C-15 are likely from molecular oxygen (10). The origin of the tricarballylic acid is generally believed to be from the citric acid cycle (7). Despite these previous biochemical studies, however, little is known about the biosynthetic sequence for these individual steps. 
Recently, a 15-gene cluster (FUM1 and FUM6-FUM19) required for the biosynthesis of fumonisins in $F$. verticillioides was cloned and characterized $(11-13)$. The identification of this cluster has facilitated direct studies of the biosynthetic genes by genetic and biochemical approaches. Among the FUM genes, FUM13 has been characterized by a genetic approach (14). Butchko et al. (14) found that a FUM13-deleted mutant accumulated the C-3 keto form of fumonisins $B_{3}$ and $B_{4}$, indicating that this gene encodes a C-3 keto reductase of fumonisins (14). More recently, Butchko et al. (15) generated a FUM3 (also FUM9) deletion mutant and showed that this gene is required for the C-5 hydroxylation of fumonisins. We also obtained biochemical data via heterologous expression in yeast that demonstrated that FUM3 encodes a 2-ketoglutarate-dependent dioxygenase that catalyzes the conversion of fumonisin $\mathrm{B}_{3}$ to $\mathrm{B}_{1}$, directly confirming that Fum $3 \mathrm{p}$ catalyzes the C-5 hydroxylation (16). Several other FUM disruption mutants, including $\triangle F U M 1, \triangle F U M 6, \triangle F U M 8, \triangle F U M 17, \triangle F U M 18$, and $\triangle F U M 19$, have also been generated by Proctor and co-workers $(11-13)$. The first three mutants did not accumulate any identifiable intermediates that could have provided additional information about the function of these genes, while the latter mutants were not or only subtly affected in their ability to produce fumonisins. Therefore, additional and alternative approaches are needed to study the functions of these genes. Here, we present data obtained from coculture experiments using three $F U M$ disruption mutants, $\triangle F U M 1, \triangle F U M 6$, and $\triangle F U M 8$. The results in this study provide evidence for the order of some of the early biochemical reactions in the fumonisin biosynthetic pathway.

\section{MATERIALS AND METHODS}

Materials and Growth of Fungi. Standard fumonisin $\mathrm{B}_{1}, \mathrm{~B}_{2}, \mathrm{~B}_{3}$, and $\mathrm{B}_{4}$ were gifts from Dr. Ronald D. Plattner (USDA, Peoria, IL) or purchased from Promec Unit, The Medical Research Council, South Africa. Corn and corn meal were purchased from a local store. Wildtype strain $F$. verticillioides A0149 was provided by Dr. Gilchrist (University of California, Davis). General fungal growth was in GYP medium (2\% glucose, $1 \%$ peptone, and $0.3 \%$ yeast extract) (11). Conidia were collected from V-8 juice agar plates after 3 weeks growth as described (11). CMK (cracked maize kernels) or GYAM medium $(0.12 \mathrm{M}$ glucose, $50 \mathrm{mM}$ malic acid, $8.0 \mathrm{mM}$ l-asparagine, $1.7 \mathrm{mM}$ $\mathrm{NaCl}, 4.4 \mathrm{mM} \mathrm{K}_{2} \mathrm{HPO}_{4}, 2.0 \mathrm{mM} \mathrm{MgSO}$, $8.8 \mathrm{mM} \mathrm{CaCl}_{2}$, and $0.05 \%$ yeast extract, adjusted to $\mathrm{pH} 3.0$ with $\mathrm{HCl}$ ) were used for fumonisin production $(10,11)$. CMK was soaked in water for $24 \mathrm{~h}$ and thoroughly washed with water prior to autoclaving to remove any potential fumonisin contamination on the kernels. No fumonisin was detected in the medium (by HPLC-ELSD and LC-MS analysis, see below).

Coculture of Mutants. Gene-disruption mutants $\triangle F U M 1$ (originally designated $\triangle F U M 5$, strain GFA2364) and $\triangle F U M 6$ (strain GFA3075) were previously generated by replacing internal fragments of FUM1 and FUM6, respectively, with the hygromycin marker gene (Hyg) (11, 13). Mutant $\triangle F U M 8$ (strain GFA3245) was also previously generated but via additive gene disruption of the FUM8 gene, which resulted in a mutant containing two incomplete copies of the FUM8 coding region (13). Approximately $8 \times 10^{7}$ conidia of mutant strains were used to inoculate CMK $(5 \mathrm{~g})$ medium or liquid GYAM $(10 \mathrm{~mL}$, containing $7 \%$ corn meal). Mycelia plugs from V8 plates were also used for inoculations in several batches. The cultures were allowed to grow for 2-3 weeks with occasional shaking to allow even distribution of the cultures. In the time-course studies, a total of eight CMK $(5 \mathrm{~g})$ cultures were inoculated with a $3: 1$ mixture of $\Delta F u m 6+\Delta F u m 5$ or $\Delta F u m 6+$ $\Delta$ Fum 8 (approximately $6 \times 10^{10}$ conidia). Two milliliters of sterilized distilled water was added to the media after inoculation. The wildtype strain and individually inoculated mutants served as controls. The cultures were harvested from the 3rd to the 7 th day by the extraction of chloroform $(10 \mathrm{~mL})$. Three duplicated samples were obtained for each time point. The extracts were dried in a rotary evaporator and redissolved in $1 \mathrm{~mL}$ of $50 \%$ methanol for analysis by HPLC-ELSD and LC-MS.

Metabolite Extraction. Initially, the cultures of mutants on CMK medium were extracted with $\mathrm{H}_{2} \mathrm{O}$ or $\mathrm{CH}_{3} \mathrm{OH} / \mathrm{CHCl}_{3}$ (1:1 v/v) separately, to get all possible metabolites accumulated in the mutants. After the metabolite pattern in the extracts was checked by HPLC analysis, cultures from $\mathrm{CMK}$ were extracted with a mixture of $\mathrm{CHCl}_{3} / \mathrm{CH}_{3} \mathrm{OH} /$ $\mathrm{H}_{2} \mathrm{O}(1: 1: 1 \mathrm{v} / \mathrm{v} / \mathrm{v})$ in all subsequent experiments. Typically, a $5 \mathrm{~g} \mathrm{CMK}$ culture was soaked in $15 \mathrm{~mL}$ of solvent overnight and filtered through Whatman no. 1 paper. The filtrate was dried in a rotary evaporator and resuspended in $2.5 \mathrm{~mL}$ of water. This solution was filtered through a $0.2 \mu \mathrm{m}$ syringe filter (Nalge Company, New York) and directly subjected to HPLC analysis. For culture from GYAM, the $10-\mathrm{mL}$ medium was filtered through Whatman no. 1 paper, and $3 \mathrm{~g}$ of Amberlite XAD-4 beads was added to the resulting solution, which was gently shaken overnight. The XAD beads were then washed with water, and metabolites were eluted from the beads with $100 \%$ methanol. The eluted solution was dried in a rotary evaporator and redissolved in water. This solution was filtered and subjected to HPLC analysis.

Analytical Methods. The high-performance liquid chromatography (HPLC) system was a ProStar, Model 210 from Varian (Walnut Creek, CA) with a $250 \times 4.6 \mathrm{~mm}$ i.d., $5 \mu \mathrm{m}$, Alltima C18LL column (Alltech, Deerfield, IL). The mobile phases were (A) water/TFA (100:0.025 v/v) and (B) acetonitrile/TFA (100:0.025 v/v) with a gradient of 0 to $20 \%$ $\mathrm{B}$ in $\mathrm{A}$ in the first $5 \mathrm{~min}, 20 \%$ to $40 \% \mathrm{~B}$ in $\mathrm{A}$ from 5 to $10 \mathrm{~min}, 40 \%$ to $80 \% \mathrm{~B}$ in A from 10 to $15 \mathrm{~min}, 80 \%$ to $80 \%$ B in A from 15 to 20 min, and $80 \%$ to $0 \% \mathrm{~B}$ in $\mathrm{A}$ from 20 to $25 \mathrm{~min}$. The flow rate was 1.0 $\mathrm{mL} / \mathrm{min}$, and a $40 \mu \mathrm{L}$ portion was injected in all analytical experiments. The conditions set for light scattering detection on an ELSD2000 (Alltech, Deerfield, IL) were $45^{\circ} \mathrm{C}$ of drift tube temperature, $2.0 \mathrm{~L} / \mathrm{min}$ of gas flow, and gain value of 1 in the impactor-on mode. For preparative HPLC, the respective fractions were collected directly from the column according to their retention times. For liquid chromatography-mass spectrometry (LC-MS), a $250 \times 1 \mathrm{~mm}$ i.d., $5 \mu \mathrm{m}, \mathrm{RFC}_{18}$ Vydac column (Vydac) was used. The solvent systems were (A) water/ formic acid (100:0.1 v/v) and (B) 95\% acetonitrile/formic acid (100/ $0.1 \mathrm{v} / \mathrm{v}$ ). The flow rate was $0.05 \mathrm{~mL} / \mathrm{min}$, and the injection volume was $50 \mu \mathrm{L}$. All positive electrospray spectra were acquired on a VG Platform II mass spectrometer or a Micromass Q-Tof (Manchester, U.K.).

\section{RESULTS AND DISCUSSION}

Analysis of Metabolites in Mutants $\triangle F U M 1, \triangle F U M 6$, and $\Delta \boldsymbol{F U M 8}$. Cultures were extracted with chloroform/methanol or water. The chloroform/methanol extracts formed two layers after centrifugation, with the upper layer being methanol, together with water from the culture medium, and the lower layer being chloroform. While the methanol layer or the water-alone extracts did not contain any obvious new peaks on HPLC-ELSD when compared to that from the wild-type strain, the chloroform layer gave approximately $8-15$ peaks $(13-17$ min retention times) on HPLC-ELSD (data not shown). Most of the peaks were common to all the mutants, suggesting that the compounds may not be directly related to the mutated genes. However, extracts from $\triangle F U M 6$ and $\triangle F U M 8$ contained $1-2$ peaks that were not present in extracts of $\triangle F U M 1$ or wild-type strain (data not shown). The results suggest that potential intermediates could be present in $\triangle F U M 6$ and $\triangle F U M 8$ mutants. The yield of the new peaks was low, and attempts to directly characterize them were not successful. To understand the role of the mutated genes in the biosynthetic pathway of fumonisins, we took an alternative approach, the coculturing of mutants.

Complementation of $\triangle F U M 6$ by Coculture with $\triangle F U M 1$ or $\boldsymbol{\Delta} \boldsymbol{F U M 8}$. HPLC analysis of extracts from wild-type $F$. verticillioides revealed one major peak with a retention time of $16.48 \mathrm{~min}$, which is the same as that of standard fumonisin $\mathrm{B}_{1}$ (Figure 2A), and 5-6 minor peaks in the same region. Among 


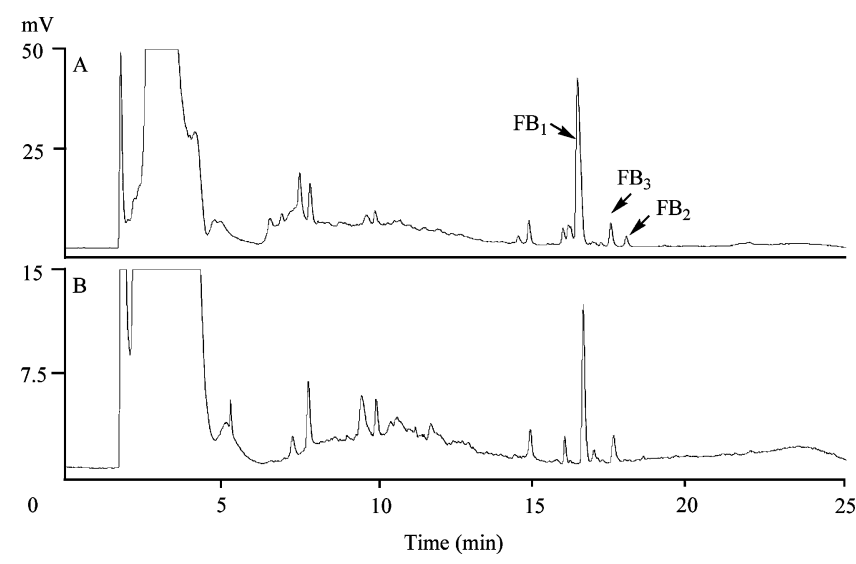

Figure 2. HPLC-ELSD analysis of extracts from 2-week-old CMK cultures. (A) Wild-type F. verticillioides; (B) coculture of mutants $\triangle F U M 1$ and $\triangle F U M 6$.

these minor peaks, two with retention times of 17.55 and 18.04 min coeluted with the fumonisin $\mathrm{B}_{3}$ and $\mathrm{B}_{2}$ standards, respectively. The identity of these peaks was confirmed by LC-MS analysis. Under the experimental conditions used, the major peak had a retention time of $8.40 \mathrm{~min}$ on LC-MS and a $[\mathrm{M}+\mathrm{H}]^{+}$of $\mathrm{m} / \mathrm{z}$ 722.8, which are the same as those of standard fumonisin $\mathrm{B}_{1}$ (data not shown). The two minor peaks had retention times of 8.72 and $8.88 \mathrm{~min}$, respectively. They have the same [M+ $\mathrm{H}]^{+}$of $m / z$ 706.9, which is the same as those of fumonisin $\mathrm{B}_{3}$ and $\mathrm{B}_{2}$ (data not shown). The estimated yield of fumonisins in the wild type was $1100-1700 \mu \mathrm{g}$ of fumonisin $\mathrm{B}_{1}, 320-410$ $\mu \mathrm{g}$ of $\mathrm{B}_{2}$, and $690-810 \mu \mathrm{g}$ of $\mathrm{B}_{3}$ per gram of CMK solid culture after 2 weeks of growth.

In contrast to cultures of individual FUM mutants, cocultures of some of the mutants produced fumonisins. According to HPLC-ELSD analysis, extracts of cocultures of the $\triangle F U M 1$ and $\triangle F U M 6$ mutants grown for 2-3 weeks in either CMK or GYAM contained metabolites that coeluted with the fumonisin $\mathrm{B}_{1}$ and $\mathrm{B}_{3}$ standards (Figure 2B). LC-MS analysis confirmed the presence of fumonisin $\mathrm{B}_{1}$ and $\mathrm{B}_{3}$ in the $\triangle F U M 1+\triangle F U M 6$ coculture (Figure $\mathbf{3 A}$ ). The estimated yield of fumonisins in the cocultures was $820-1100 \mu \mathrm{g}$ of $\mathrm{B}_{1}, 170-190 \mu \mathrm{g}$ of $\mathrm{B}_{2}$, and $310-370 \mu \mathrm{g}$ of $\mathrm{B}_{3}$ per gram of CMK solid culture or 130$140 \mu \mathrm{g}$ of $\mathrm{B}_{1}, 31-39 \mu \mathrm{g}$ of $\mathrm{B}_{2}$, and $68-81 \mu \mathrm{g}$ of $\mathrm{B}_{3}$ per milliliter of GYAM liquid culture, after 2 weeks of growth. A similar result was obtained when the $\triangle F U M 6$ and $\triangle F U M 8$ mutants were cocultured (Figure 3C) but not when the $\triangle F U M 1$ and $\triangle F U M 8$ mutants were cocultured (Figure 3B). Thus, fumonisin production was restored only in cocultures that included the $\triangle F U M 6$ mutant.

The coculture results implied that a putative intermediate(s) produced by the $\triangle F U M 6$ mutant was secreted into the culture medium, taken up by $\triangle F U M 1$ or $\triangle F U M 8$ mutant, and subsequently metabolized to fumonisins. This process did not occur in cocultures of the $\triangle F U M 1$ and $\triangle F U M 8$ mutants. The results suggest that the blockage of the biosynthetic pathway at the FUM1 and FUM8 stage does not result in the accumulation of intermediates. FUMI encodes a polyketide synthase catalyzing the assembly of the carbon backbone of fumonisins (11). According to the paradigm of polyketide synthase-catalyzed reactions, all intermediates will be covalently attached to the enzyme throughout the entire process of chain elongation (17). A disruption of the polyketide synthase gene (FUM1) would result in no carbon chain assembly, whereas a disruption of a step immediately downstream of the polyketide synthase would result in the accumulation of the covalently bound carbon chains.
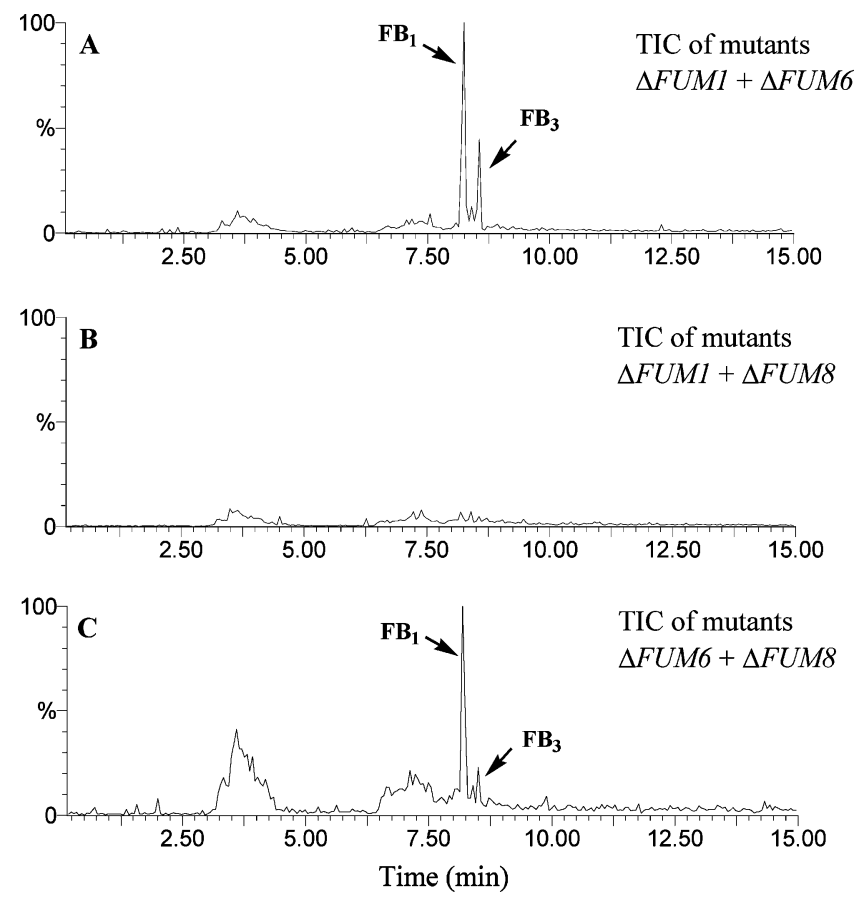

Figure 3. LC-MS analysis of extracts from 2-week-old CMK cocultures of mutants. (A) Total ion current (TIC) of mutants $\triangle F U M 1$ and $\triangle F U M 6$ coculture; (B) TIC of mutants $\triangle F U M 1$ and $\triangle F U M 8$ coculture; (C) TIC of mutants $\triangle F U M 6$ and $\triangle F U M 8$ coculture.

Normally, such bound intermediates could not be released into the media unless a thioesterase domain (or a functional equivalent) is present in the polyketide synthase (17). Fum1p does not have such a chain-releasing domain (11). FUM8 encodes a serine palmitoyltransferase-like enzyme, which is the obvious candidate for catalyzing the condensation between alanine and the polyketide carbon chain (dimethyl stearate) of fumonisins. Furthermore, it is possible that the Fum8p includes a polyketide chain-releasing mechanism (see below). FUM6 encodes a cytochrome P450-dependent oxygenase, which is likely involved in the modification of the carbon backbone of fumonisins. The modification of a polyketide chain could take place before or after its release from the synthase $(18,19)$. Because fumonisins were produced in cocultures only when the $\triangle F U M 6$ mutant was present, the data support that the Fum8pcatalyzed condensation of dimethyl stearate and alanine precedes the Fum6p-catalyzed oxidation, i.e., the chain release precedes the chain modification. Therefore, the biosynthetic sequence in these early steps of fumonisin pathway is likely to be Fum1pFum8p-Fum6p.

Potential Intermediates. To further understand the nature of the putative intermediates accumulated in $\triangle F U M 6$, we carried out a series of feeding experiments. We envisage that the feeding of the intermediate compounds isolated from the $\triangle F U M 6$ mutant to the $\triangle F U M 1$ or $\triangle F U M 8$ mutant could result in the fumonisin production. However, feeding experiments using the total extract as well as the individual HPLC fractions failed to produce consistent results, suggesting that the potential intermediates could be very unstable when isolated. Because the coculture experiments consistently produced fumonisins, $\triangle F U M 6$ must produce one or more intermediates. We speculated that if we followed a time course of the $\triangle F U M 6$ and $\triangle F U M 1$ coculture or $\triangle F U M 6$ and $\triangle F U M 8$ coculture, we would be able to detect an intermediate(s). According to HPLC-ELSD analysis, beginning 3 days after the start of the $\triangle F U M 6$ and $\triangle F U M 1$ coculture or $\triangle F U M 6$ and $\triangle F U M 8$ coculture, $2-3$ new peaks appeared 

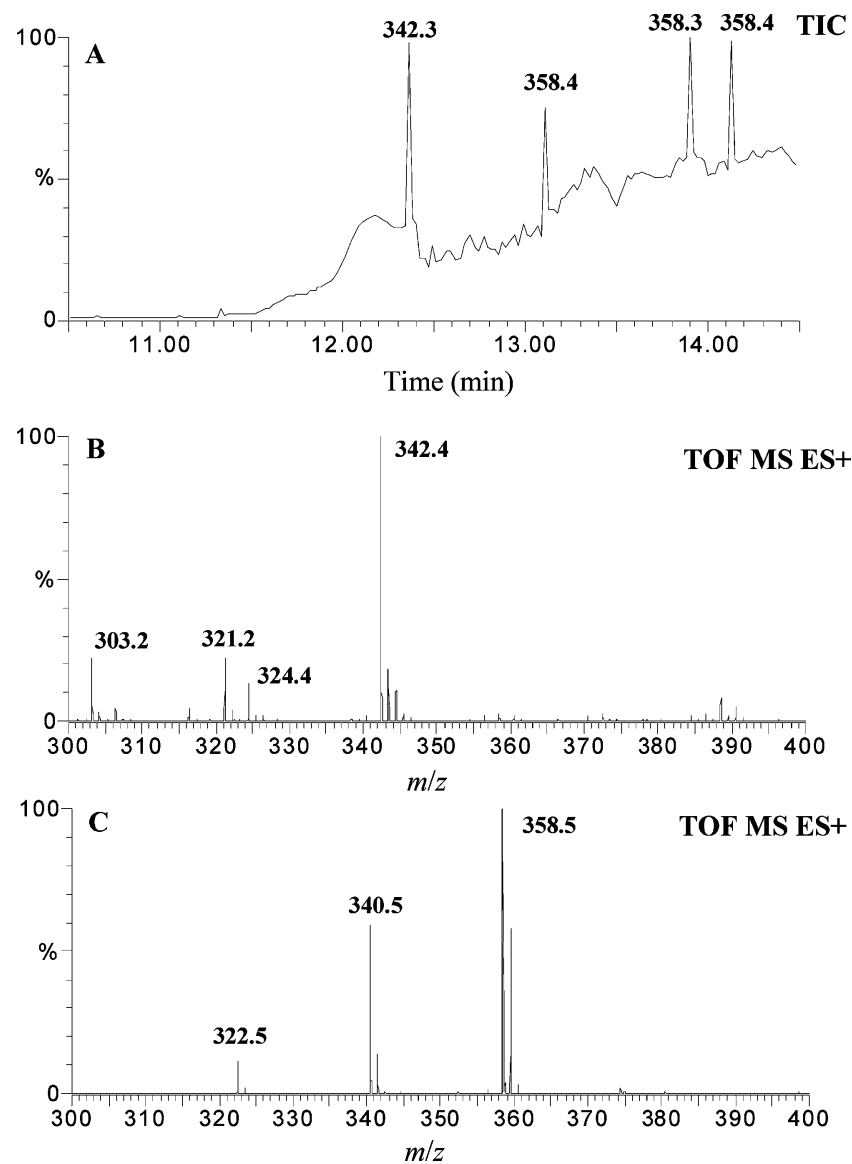

Figure 4. LC-MS analysis of the putative intermediates that accumulated in the cocultures of mutants. Extracts from the $\triangle F U M 6$ and $\triangle F U M 1$ coculture were analyzed by HPLC-ELSD over a 7-day time course, and the regions that showed decreased or increased peak intensities on HPLCELSD were collected for LC-MS analysis. (A) Total ion current (TIC) of extract from the $\triangle F U M 6$ and $\triangle F U M 1$ coculture at the 7 th day; $(B)$ electrospray mass spectrum time-of-flight (TOF) analysis of the peak at 12.4 min in panel $\mathbf{A} ;(\mathbf{C})$ electrospray mass spectrum TOF analysis of the peak at $13.1 \mathrm{~min}$ in panel $\mathbf{A}$.

at retention times of 17-20 min. More interestingly, the intensity of some of these peaks gradually decreased over the time course, whereas others gradually increased (data not shown). We subsequently collected the peaks at this region from HPLC and analyzed them by LC-MS. The results showed that four major peaks were present in the 10-15 min region (Figure 4A). The peak at $12.4 \mathrm{~min}$ gave a $[\mathrm{M}+\mathrm{H}]^{+}$of $\mathrm{m} / \mathrm{z} 342.3$, whereas the peaks at $13.1,13.9$, and $14.1 \mathrm{~min}$ had the same $[\mathrm{M}+\mathrm{H}]^{+}$of $\mathrm{m} / \mathrm{z}$ 358.4. An analysis of the mass spectra of the peak at 12.4 min (Figure 4B) and $13.1 \mathrm{~min}$ (Figure 4C) suggested that 1-2 hydroxyl groups are likely present in the molecules. A loss of one hydroxyl group (as a water molecule) from the $\mathrm{m} / \mathrm{z}, 342.4$ ion would give rise to the $\mathrm{m} / \mathrm{z}, 324.4$ ion in Figure 4B, and a loss of one or two hydroxyl groups from the $\mathrm{m} / \mathrm{z} 358.5$ ion would account for the $m / z, 340.5$ or 322.5 ion, respectively, in Figure 4C. We further examined the peaks using MS-MS analysis. The results not only confirmed the presence of hydroxyl groups in the molecules but also indicated the presence of a long aliphatic chain in the molecules as evidenced by the characteristic fragmentation pattern of peaks with a 14 mass-unit difference (for the $\mathrm{CH}_{2}$ groups) (data not shown). Minor peaks in this region gave $[\mathrm{M}+\mathrm{H}]^{+}$of $\mathrm{m} / \mathrm{z} 340.3,374.4$, and 390.4 (data not shown). These masses are coincident with those of compounds that have the general carbon skeleton of fumonisins but with various numbers of hydroxyl groups (Figure 5). The first major peak has a $[\mathrm{M}+\mathrm{H}]^{+}$of $m / z, 342.3$, which is the expected mass for the 3-ol product (2-amino-3-hydroxy-12,16-dimethylicosane) (Figure 5). This product could be generated by a condensation between the putative 20-carbon polyketide and alanine followed by reduction of the 3-keto function to 3-ol. The putative 3-keto compound (2-amino-3-oxo-12,16-dimethylicosane) has a calculated mass of 339.6. Only a minor peak of $[\mathrm{M}+\mathrm{H}]^{+}$of $\mathrm{m} / \mathrm{z}$ 340.3 was found in the samples, suggesting that the 3-keto product is readily reduced by a reductase, presumably Fum $13 \mathrm{p}$ (14), in the $\triangle F U M 6$ mutant. The $[\mathrm{M}+\mathrm{H}]^{+}$of $\mathrm{m} / z, 358.4$ of the other three major peaks is consistent with the addition of a second hydroxyl group to the 3-ol product to form diol products. Since they had the same mass but different retention times, the diol products are likely to be isomers resulting from regioselective (e.g., 14-ol versus 15-ol) and/or stereoselective (e.g., epimers) hydroxylations. Finally, the presence of minor products with $[\mathrm{M}+\mathrm{H}]^{+}$of $\mathrm{m} / z 374.4$ and 390.4 suggests that a third and fourth hydroxyl group were added to the carbon backbone to produce triol and tetraol products, respectively (see Figure 5 for structures).

The identification of the putative fumonisin intermediates in the $\triangle F U M 6$ and $\triangle F U M 1$ coculture and $\triangle F U M 6$ and $\triangle F U M 8$ coculture has significant implications in the understanding of the fumonisin biosynthetic pathway. First, a compound with a single carbon-carbon bond between C-14 and C-15 is probably the direct substrate for the Fum6p-catalyzed hydroxylation. It was previously implied that the vicinal diol at C-14 and C-15 could be generated by epoxide hydrolation (20). If this were the case, Fum6p would catalyze an epoxidation at C-14 and $\mathrm{C}-15$, and this would require a substrate with a double bond between $\mathrm{C}-14$ and $\mathrm{C}-15$. Moreover, opening the epoxide ring to generate the vicinal diol would also require an epoxide hydrolase. However, no epoxide hydrolase gene was found in the FUM gene cluster (12). Because the four major peaks present in the coculture do not suggest the presence of a double bond or a vicinal diol at C-14 and C-15, our results provide evidence for a direct hydroxylation of a saturated carbon chain, rather than a ring opening of an epoxide intermediate. Second, the coexistence of multiple components with the same mass for the diol compounds suggests that the oxidation enzymes may not have a strict substrate specificity. In addition to FUM6, there are two other P450 genes (FUM12 and FUM15) found in the FUM gene cluster (12). The latter two P450 enzymes may be responsible for the addition of the third hydroxyl group (e.g., in 3,14,15-triol product) or the fourth hydroxyl group (e.g., in 3,10,14,15-tetraol product) (Figure 5). Although the exact regiospecificity of $\mathrm{P} 450$ enzymes is not clear, our data suggest that the oxidation steps could take place at various stages in the biosynthesis of fumonisins.

The cloning of the FUM gene cluster and successful isolation of biosynthetic intermediates from mutants $\triangle F U M 9$ and $\triangle F U M 13$ have provided important information for the biosynthetic mechanism of fumonisins $(11-15)$. FUM3 encodes a dioxygenase that catalyzes the $\mathrm{C}-5$ hydroxylation of fumonisins ( 15 , 16), while FUM13 encodes a keto reductase that catalyzes the reduction of C-3 carbonyl to a hydroxyl (14). Only derivatives of fumonisin $\mathrm{B}_{3}$ and $\mathrm{B}_{4}$, but not fumonisin $\mathrm{B}_{1}$ and $\mathrm{B}_{2}$, accumulated in $\triangle F U M 3$ and $\triangle F U M 13$ mutants. This indicates that the steps catalyzed by these two enzymes may occur later in the biosynthetic pathway or that downstream enzymes are able to process at least some "unnatural" substrates (e.g., the C-3 keto form) to fumonisin $\mathrm{B}_{3}$ and $\mathrm{B}_{4}$. In this study, we have successfully applied the coculture approach to obtain biosyn- 

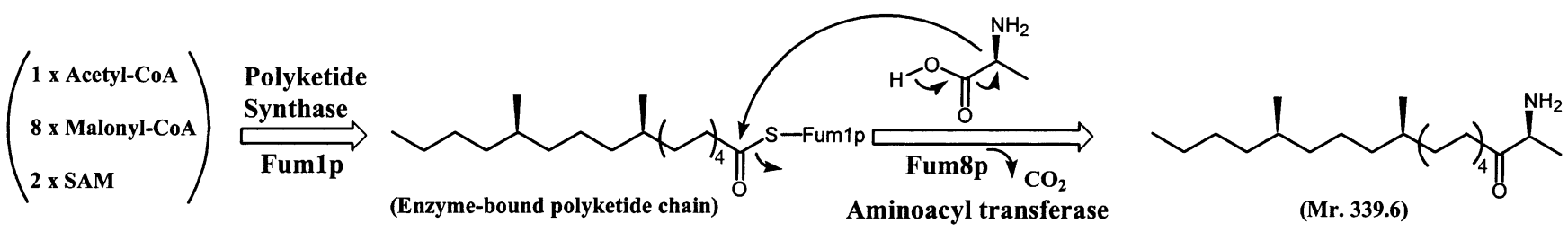

(Polyketide-alanine condensation product)

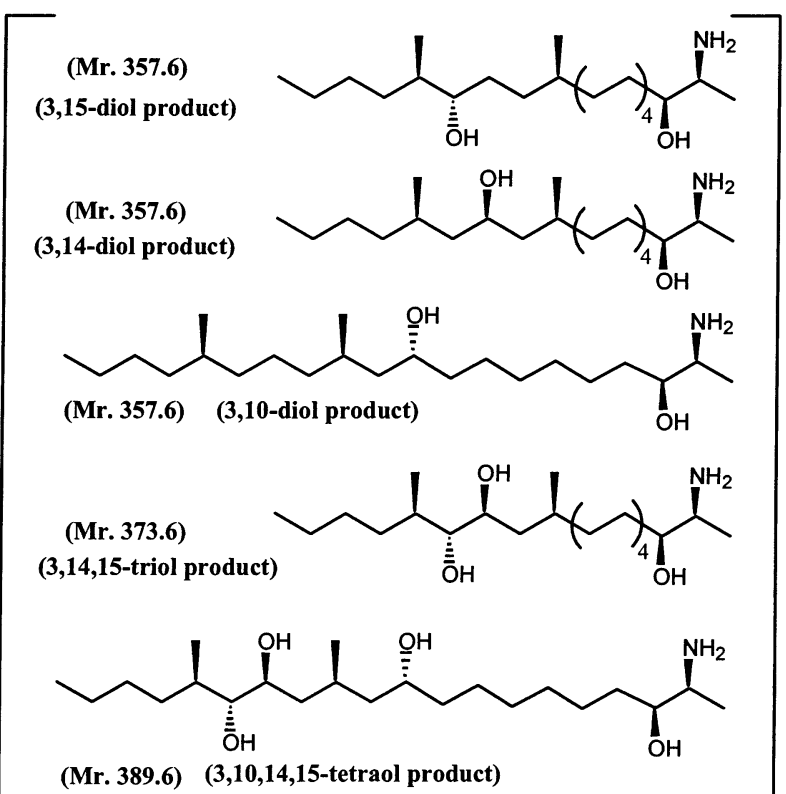

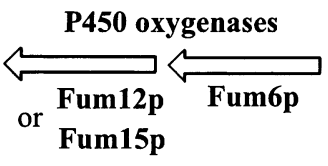

Precursor from TCA cycle

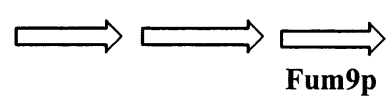

Figure 5. Proposed biosynthetic pathway for fumonisins. Molecular weights for putative intermediates were included to account for the LC-MS data observed in Figure 4. For the structure of fumonisins $\mathrm{B}_{1}, \mathrm{~B}_{2}, \mathrm{~B}_{3}$, and $\mathrm{B}_{4}$, see Figure 1. SAM, S-adenosylmethionine.

thetic information from mutants that do not accumulate any isolatable intermediate. By following a time course of coculture, rather than an end product of individual culture of the mutants, we were able to identify a number of putative intermediates. The data provide evidence for the sequence of enzymatic steps in fumonisin biosynthesis. On the basis of these data and those from previous studies $(11-16)$, we propose a biosynthetic pathway as depicted in Figure 5. The biosynthesis starts with the Fum1p-catalyzed carbon chain assembly from one molecule of acetyl CoA, eight molecules of malonyl CoA, and two molecules of methionine (in $S$-adenosyl form). The $\mathrm{C}_{18}$ polyketide chain is released from the enzyme by a nucleophilic attack of a carbanion, which is derived from $\alpha$-carbon of alanine by decarboxylation, on the carbonyl carbon of polyketide acyl chain. This step is catalyzed by a pyridoxal 5'-phosphate- (PLP-) dependent aminoacyl transferase Fum8p $(13,14)$. The resultant 3-keto intermediate is then stereospecifically reduced to a 3-hydroxyl product by reductase Fum13p (14). Subsequent oxidations at $\mathrm{C}-14$ and $\mathrm{C}-15$ positions by cytochrome $\mathrm{P} 450$ dependent oxygenases Fum6p, Fum12p, or Fum15p, tricarballylic esterification of the hydroxyl groups on C-14 and C-15 by acyltransferase Fum 14p, and C-5 hydroxylation by 2-ketoglutarate-dependent dioxygenase Fum $3 p$ furnish the biosynthesis of fumonisins (Figure 5). The tricarballylic moieties are most likely derived from the citric acid cycle (e.g., aconitic acid) (7), and their addition to the carbon backbone may involve Fum7p, Fum10p, Fum11p, and Fum14p (Butchko, Plattner, and Proctor, unpublished data). A reduction of aconitate by Fum7p followed by a thioesterification with $\mathrm{CoA}$ as cosubstrate, catalyzed by Fum10p, would produce an activated acyl-S-CoA of tricarballylic acid. Fum $14 p$ then catalyzes the transfer of tricarballylic acyl-CoA to the diols on C-14 and C-15 to form the diesters of fumonisins. The validation of the sequence of latter reactions in the fumonisin biosynthetic pathway will require further studies with both genetic and biochemical approaches.

\section{ACKNOWLEDGMENT}

We thank Ronald D. Plattner (USDA, Peoria, IL) for providing standard fumonisins and David G. Gilchrist (University of California, Davis, CA) for $F$. verticillioides wild-type strain A0149. We are grateful to Dr. Kurt Wulser (Nebraska Center for Mass Spectrometry, University of Nebraska-Lincoln) for technical assistance in LC-MS analysis.

\section{LITERATURE CITED}

(1) Marasas, W. F. Discovery and occurrence of the fumonisins: A historical perspective. Environ. Health Perspect. 2001, 109 (Suppl. 2), 239-243.

(2) Nelson, P. E.; Desjardins, A. E.; Plattner, R. D. Fumonisins, mycotoxins produced by Fusarium species: Biology, chemistry, and significance. Annu. Rev. Phytopathol. 1993, 31, 233-252.

(3) Desjardins, A. E.; Plattner, R. D. Fumonisin B(1)-nonproducing strains of Fusarium verticillioides cause maize (Zea mays) ear infection and ear rot. J. Agric. Food Chem. 2000, 48, 57735780.

(4) Gelderblom, W. C.; Jaskiewicz, K.; Marasas, W. F.; Thiel, P. G.; Horak, R. M.; Vleggaar, R.; Kriek, N. P. Fumonisins-novel mycotoxins with cancer-promoting activity produced by Fusarium moniliforme. Appl. Environ. Microbiol. 1988, 54, 18061811.

(5) Marasas, W. F.; Kellerman, T. S.; Gelderblom, W. C.; Coetzer, J. A.; Thiel, P. G.; van der Lugt, J. J. Leukoencephalomalacia in a horse induced by fumonisin B1 isolated from Fusarium moniliforme. Onderstepoort J. Vet. Res. 1988, 55, 197-203. 
(6) Bezuidenhout, S. C.; Gelderblom, W. C. A.; Gorst-Allman, C. P.; Horak, R. M.; Marasas, W. F. O.; Spiteller, G.; Vleggaar, R. Structure elucidation of the fumonisins, mycotoxins from Fusarium moniliforme. J. Chem. Soc., Chem. Commun. 1988, 743-745.

(7) Blackwell, B. A.; Edwards, O. E.; Fruchier, A.; ApSimon, J. W.; Miller, J. D. NMR structural studies of fumonisin B1 and related compounds from Fusarium moniliforme. Adv. Exp. Med. Biol. 1996, 392, 75-91.

(8) Branham, B. E.; Plattner, R. D. Alanine is a precursor in the biosynthesis of fumonisin B1 by Fusarium moniliforme. Mycopathologia 1993, 124, 99-104.

(9) Plattner, R. D.; Shackelford, D. D. Biosynthesis of labeled fumonisins in liquid cultures of Fusarium moniliforme. Mycopathologia 1992, 117, 17-22.

(10) Caldas, E. D.; Sadilkova, K.; Ward, B. L.; Jones, A. D.; Winter, C. K.; Gilchrist, D. G. Biosynthetic studies of fumonisin B1 and AAL toxins. J. Agric. Food Chem. 1998, 46, 4734-4743.

(11) Proctor, R. H.; Desjardins, A. E.; Plattner, R. D.; Hohn, T. M. A polyketide synthase gene required for biosynthesis of fumonisin mycotoxins in Gibberella fujikuroi mating population A. Fungal Genet. Biol. 1999, 27, 100-112.

(12) Proctor, R. H.; Brown, D. W.; Plattner, R. D.; Desjardins, A. E. Co-expression of 15 contiguous genes delineates a fumonisin biosynthetic gene cluster in Gibberella moniliformis. Fungal Genet. Biol. 2003, 38, 237-249.

(13) Seo, J. A.; Proctor, R. H.; Plattner, R. D. Characterization of four clustered and coregulated genes associated with fumonisin biosynthesis in Fusarium verticillioides. Fungal Genet. Biol. 2001, 34, 155-165.

(14) Butchko, R. A.; Plattner, R. D.; Proctor, R. H. FUM13 encodes a short chain dehydrogenase/reductase required for $\mathrm{C}-3$ carbonyl reduction during fumonisin biosynthesis in Gibberella moniliformis. J. Agric. Food Chem. 2003, 51, 3000-3006.
(15) Butchko, R. A. E.; Plattner, R. D.; Proctor, R. H. FUM9 is required for $\mathrm{C}-5$ hydroxylation of fumonisins and complements the mitotically defined Fum3 locus in Gibberella moniliformis. Appl. Environ. Microbiol. 2003, 69, 6935-6937.

(16) Ding, Y.; Bojja, R. S.; Du, L. Fum3p is a 2-ketoglutarate dependent dioxygenase required for $\mathrm{C}-5$ hydroxylation of fumonisins in Fusarium verticillioides. Appl. Environ. Microbiol. 2004, in press.

(17) Du, L.; Shen, B. Biosynthesis of hybrid peptide-polyketide natural products. Curr. Opin. Drug Discov. Devel. 2001, 4, 215228.

(18) Staunton, J.; Weissman, K. J. Polyketide biosynthesis: A millennium review. Nat. Prod. Rep. 2001, 18, 380-416.

(19) Kennedy, J.; Auclair, K.; Kendrew, S. G.; Park, C.; Vederas, J. C.; Hutchinson, C. R. Modulation of polyketide synthase activity by accessory proteins during lovastatin biosynthesis. Science 1999, 284, 1368-1372.

(20) Morisseau, C.; Ward, B. L.; Gilchrist, D. G.; Hammock, B. D. Multiple epoxide hydrolases in Alternaria alternata f. sp. lycopersici and their relationship to medium composition and host-specific toxin production. Appl. Environ. Microbiol. 1999, $65,2388-2395$.

Received for review December 4, 2003. Revised manuscript received March 4, 2004. Accepted March 5, 2004. This study was supported in part by a faculty startup fund from University of Nebraska-Lincoln and Faculty Seed Grant from Nebraska Research Council. The mass spectrometry facility is supported in part by NIH Grant P20 RR 15635 from the COBRE Program of the National Center for Research Resources, NCI Cancer Center Support Grant P30 CA36727, and the Nebraska Research Initiative.

JF035429Z 